\title{
On-chip quantum teleportation
}

\author{
B. J. Metcalf, N. Thomas-Peter, J. B. Spring, P. C. Humphreys, N. K. Langford, \\ W. S. Kolthammer, M. Barbieri, X. -M. Jin, B. Smith, I. A. Walmsley \\ Clarendon Laboratory, University of Oxford, Oxford, OX1 3PU, UK
}

J. C. Gates, D. Kundys, P. G. R. Smith

Optoelectronics Research Centre, University of Southampton, Southampton, SO17 1BJ, UK

Integrated quantum optics provides great promise for enabling photonic experiments to reach new regimes of complexity. Chip-based fabrication enables sophisticated networks involving multiple interfering pathways in a compact and stable physical architecture. In a recent work [1] we demonstrated an advance in the complexity of these integrated devices by showing the first quantum interference of three separate input photons. Such complexity allows the demonstration of three qubit protocols never before realised on-chip. Here, we report the first results showing quantum teleportation on an integrated photonic circuit. Quantum teleportation provides the mechanism for quantum relay schemes and is an important constituent in linear optical quantum computing. Whilst demonstrations to date have relied upon complex and fragile bulk optical systems, being able to teleport quantum states on a compact and stable integrated architecture will be a critical step towards scalable realisations of these schemes.

The circuit used here is shown schematically in figure 1(a) and is fabricated using a UV direct-write technique on a silica-on-silicon platform [2]. Three identical photons are generated via two spontaneous parametric down-conversion sources which are encoded into three quantum bits using a dual-rail encoding scheme. Thermo-optic heaters integrated onto the chip allow for the on-chip preparation of different quantum states. A successful teleportation event is recorded when a photon arrives in each of the three qubit rails. This results in the quantum state of Alice's photon being teleported onto Bob's photon. The success of the protocol is confirmed using on-chip quantum state tomography and maximum likelihood reconstruction.
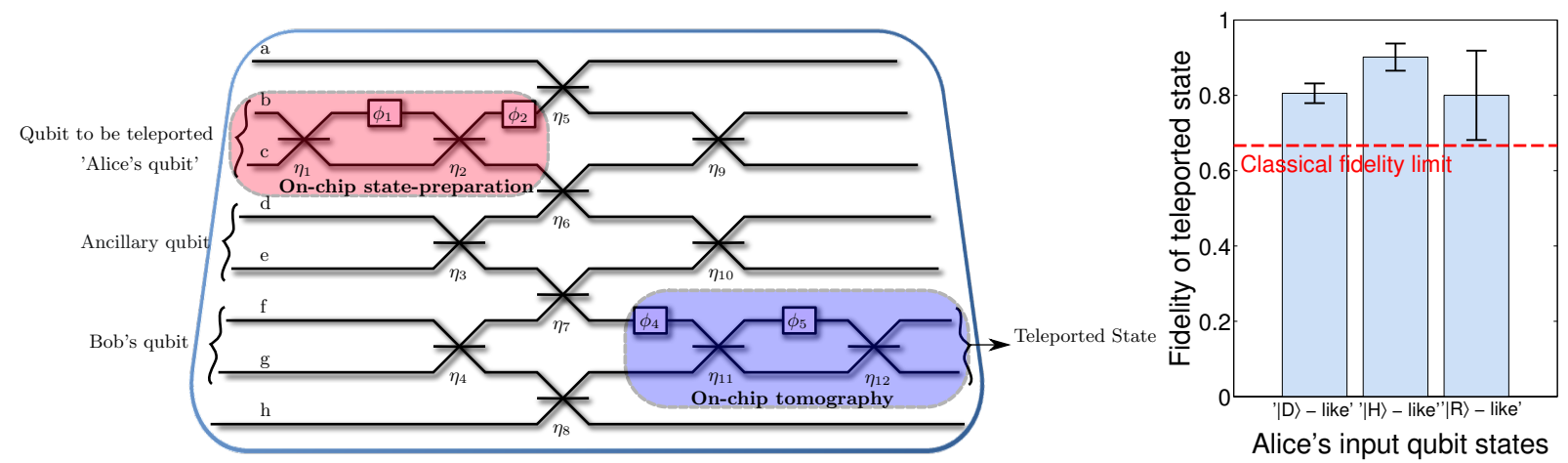

Fig. 1: (a) Schematic of the fabricated photonic circuit. The circuit operates with three input photons which define Alice's qubit, an ancillary qubit and Bob's qubit in a dual-rail encoding scheme. Beam-splitters $\eta_{1}-\eta_{4}$ and $\eta_{9}-\eta_{12}$ are designed with $1 / 2$ reflectivity whilst all others are designed to have 1/3 reflectivity. (b) Results of on-chip teleportation for three input states.

The results are summarised in figure 1(b) which shows the measured fidelity of three teleported states to those of the three prepared input states. Each state clearly beats the maximum fidelity expected from the best possible classical protocol. The three input states are prepared as close to the three orthogonal axes of the Bloch sphere as the chip allows. We shall present details of the experiment and discuss routes towards achieving higher fidelity on-chip teleportation.

\section{References}

1. Metcalf, B. J. et al. Multiphoton quantum interference in a multiport integrated photonic device. Nature Communications 4, 1356 (2013).

2. Smith, B., Kundys, D., Thomas-Peter, N. L., Smith, P. \& Walmsley, I. A. Phase-controlled integrated photonic quantum circuits. Optics Express 17, 13516-13525 (2009). 